\title{
COMPARISON BETWEEN EXPERIMENTS AND A MULTIBODY WEAKLY NONLINEAR POTENTIAL FLOW APPROACH FOR MODELING OF MARINE OPERATIONS
}

\author{
Pierre-Yves Wuillaume \\ Ecole Centrale de Nantes / CNRS \\ INNOSEA \\ Nantes, France
}

\author{
Pierre Ferrant \\ Aurélien Babarit \\ François Rongère \\ Ecole Centrale de Nantes / CNRS \\ Nantes, France
}

\author{
Mattias Lynch \\ Adrien Combourieu \\ INNOSEA \\ Nantes, France
}

\begin{abstract}
This paper presents validation tests for a new numerical tool for the numerical simulation of marine operations. It involves multibody dynamics modeling, wave-structure interactions with large amplitude body motion and cable's dynamic modeling. Hydrodynamic loads are computed using the WS_CN weakly nonlinear potential flow solver, based on the weak-scatterer hypothesis. Large deformation of the wetted body surfaces can be taken into account. Firstly the ECN's $W S \_C N$ solver capabilities are extended to multibody simulations. A first validation test is performed by comparing numerical results to the experimental data of [1]. Then, a second validation test is proposed. It consists in the ballasting operation of a spar. The experimental set-up is described.
\end{abstract}

\section{KEYWORDS}

Marine operations, numerical modeling, multibody dynamics, potential flow theory, weak-scatterer

\section{NOMENCLATURE}

A Amplitude of the incoming regular wave;

$A_{p m} \quad$ Amplitude of the harmonic motion of Body 2;

$\boldsymbol{C}=\left(\begin{array}{ll}C_{1}^{T} & C_{2}^{T}\end{array}\right)^{T}$ Inertia and external loads;

CD Matrix of influence coefficients;

CS Matrix of influence coefficients;

$\boldsymbol{F}_{\boldsymbol{j}}$ Hydrodynamic forces on the $j^{\text {th }}$ body;

$G \quad$ Green's function;

g Gravity constant;

$H=\left(\begin{array}{ll}H_{11} & H_{12} \\ H_{21} & H_{22}\end{array}\right)$ Multibody inertia matrix;

h Water depth;

$\mathrm{k} \quad$ Wave number of the incoming regular wave;
$\boldsymbol{M}_{\boldsymbol{j}} \quad$ Hydrodynamic moments at the center of gravity on the $j^{\text {th }}$ body;

$N \quad$ Number of immerged or floating bodies in $W S_{-} C N$;

$N_{j} \quad$ Number of nodes in the mesh of the $j^{\text {th }}$ body;

$\boldsymbol{n}$ Normal vector pointing outwards the fluid domain;

$p \quad$ Pressure;

$Q_{j} \quad$ Extra discretized derived terms of the body boundary condition for the $2^{\text {nd }} \mathrm{BVP}$;

$\ddot{q} \quad$ Articular accelerations;

$S_{B_{j}} \quad$ Wetted surface of the $j^{\text {th }}$ body;

$t \quad$ Time;

$\boldsymbol{V} \quad$ Fluid velocity field;

${ }^{0} \dot{\boldsymbol{V}}_{\mathbf{0}} \quad$ Acceleration of the base with respect to $\Sigma_{e}$ and projected in its frame;

$\boldsymbol{v}_{\boldsymbol{m} \text { esh }}$ Free surface node velocity;

$\boldsymbol{v}_{\boldsymbol{j}} \quad$ Velocity of the center of gravity of the $j^{\text {th }}$ body;

$\boldsymbol{x} \quad$ Position of the control point in $\Sigma_{e}$;

$\boldsymbol{x}_{\boldsymbol{j}} \quad$ Position of the center of gravity of the $j^{\text {th }}$ body in $\Sigma_{e}$.

$\boldsymbol{\Gamma} \quad$ Internal loads;

$\eta \quad$ Total wave elevation;

$\eta^{I} \quad$ Incident wave elevation;

$\eta^{P} \quad$ Scattered wave elevation;

$\lambda \quad$ Wave length of the incoming regular wave;

$v \quad$ Damping coefficient of the absorbing beach;

$\rho \quad$ Fluid density;

$\Sigma_{e} \quad$ Inertial earth fixed reference frame;

$\phi \quad$ Total velocity potential;

$\phi^{I} \quad$ Incident velocity potential;

$\phi^{P} \quad$ Scattered velocity potential;

$\omega \quad$ Frequency of the incoming regular wave;

$\boldsymbol{\omega}_{j} \quad$ Angular velocity of the $j^{\text {th }}$ body;

$\omega_{p m} \quad$ Frequency of the harmonic motion of Body 2. 
$(*)_{n} \quad$ Normal-derivative;

$(\dot{*})$ Time-derivative;

$\frac{D_{0}(*)}{D t} \quad$ Lagrangian Time-derivative;

\section{INTRODUCTION}

For the development of the offshore wind industry, the simulation of marine operations for the installation of wind turbines is required in order to optimize procedures and reduce costs. It involves multibody dynamics modeling including multiple floating bodies, wave-structure interactions with large amplitude body motions and cable's dynamic modeling. Norms have been published by DNV on this topic [2]. They are based on simplifications (characteristic quantities, regular design wave) and safety factors. Commercial tools such as Orcaflex [3], Deeplines [4], AnySim [5] or Simo [6] are frequently used for the design of marine operations such as installation of marine energy systems. They are based on frequency-domain linear potential flow theory-based code and/or Morison equations to compute the hydrodynamic loads. The linear potential flow theory assumes small amplitude motions of the floaters and small steepness incoming waves. It also assumes that the computational domain (mesh of the floating bodies and the free surface) is stationary. Furthermore, only steady hydrodynamic loads are evaluated because of the frequencydomain approach. This approach may miss some important unsteady effects. This is the case, for instance, when two floating bodies have a large relative motion or if a body goes across the free surface or any situation with an important modification of the wetted bodies' surfaces.

Other solutions than linear potential flow theory exist. For example, the use of a time-domain body-exact theory is possible. Watai [7] coupled this theory with a remeshing algorithm and a free surface interpolation algorithm to take into account the modification of the relative positions of several floating bodies. The approach is limited to waves with small steepness. Gilloteaux [8] used a nonlinear Froude-Krylov approach in order to improve the computation of hydrodynamic loads in conditions with large amplitude waves. Nonlinear hydrostatics and Froude-Krylov loads are assumed prevailing and computed on the exact wetted body surface while a linear potential flow solver calculates the diffraction-radiation loads. Unfortunately this method is not mathematically consistent as hydrodynamic loads are not computed on the same wetted surfaces. It leads to large uncertainties on its domain of validity. Hannan [9] developed a model based on a fully nonlinear potential flow solver to simulate a lowering operation. This kind of hydrodynamic solver, although more accurate than the linear one, has limitations and may present numerical challenges. Free surface boundary equations are written on the exact free surface taking into account the perturbed waves. The CPU time is also much greater compared to a linear approach. A fourth approach is the weak-scatterer method. It was proposed by Pawlowski [10] in 1991. It assumes that the perturbation wave field generated by the floating bodies is small compared to the incident wave field, such as the free surface conditions can be linearized at the incident wave elevation level. Thus, the free surface mesh is known at each time as it corresponds to the incident wave. This method allows taking into account unsteady hydrodynamic loads and is bodyexact (no approximation on the amplitude of the motion of the floating bodies).

The LHEEA research department of Ecole Centrale de Nantes has developed a hydrodynamic solver based on this approach since 2012. It is called WS_CN. Letournel [11] developed a version for a single submerged body with translational motions. Chauvigné [12] extended it to a single surface-piercing body.

To simulate marine operations, this code has been coupled with InWave [13], a multibody solver to model articulated kinematic trees. InWave has been developed by LHEEA and INNOSEA [14]. The coupling allows the multibody dynamics and unsteady hydrodynamic loads to be taken into account without being limited by the small amplitude motions assumptions for the floating bodies.

The first objective of this paper is to validate the extension of $W S_{-} C N$ to the simulations of multiple interacting floating bodies with large relative motion amplitude. Comparison is made to the experimental results of Watai et al. [1]. The second objective is to validate the coupling with InWave by comparing numerical results to experimental results obtained at LHEEA for the case of the ballasting of a spar.

\section{PART I: VALIDATION OF THE EXTENSION TO MULTIBODY SIMULATION}

\section{Governing equations}

The fluid is assumed incompressible and inviscid whereas the flow is considered as irrotational. Thereby, the fluid problem is solved in the frame of the potential flow theory. The velocity field results from a scalar velocity potential:

$$
\boldsymbol{V}=\boldsymbol{\nabla} \phi
$$

The velocity potential, respectively the wave elevation, is decomposed as an unknown scattered (perturbation) component $\phi^{P}$ and a known incident component $\phi^{I}$, respectively $\eta^{I}$ and $\eta^{P}$ :

$$
\left\{\begin{array}{c}
\phi=\phi^{I}+\phi^{P} \\
\eta=\eta^{I}+\eta^{P}
\end{array}\right.
$$

The incident wave field is defined by:

$$
\phi^{I}=\frac{A g}{\omega} \frac{\cosh (k(z+h))}{\cosh (k h)} \sin (k x-\omega t)
$$

Where $A, g, \omega$ and $k$ represent the wave amplitude, the gravity constant, the wave frequency and the wave number. 
The incident and perturbed components follow the weakscatterer approximation:

$$
\left\{\begin{array}{l}
\phi^{P} \ll \phi^{I} \\
\eta^{P} \ll \eta^{I}
\end{array}\right.
$$

$\phi^{P}$ satisfies the Laplace equation in the fluid domain:

$$
\Delta \phi^{P}=0
$$

By using the Green's second identity, one can write the well-known integral equation:

$$
\begin{gathered}
\phi^{P}(M) \Omega(M)+\iint_{S} \phi_{n}^{P}(P) G(M, P) d S \\
-\iint_{S} G_{n}(M, P) \phi^{P}(P) d S=0
\end{gathered}
$$

Where $G$ is the Rankine source distribution:

$$
G(M, P)=\frac{1}{|M P|}
$$

Equation (6) can be discretized. It yields:

$$
\operatorname{CS} \phi_{n}^{P}-\operatorname{CD} \phi^{P}=0
$$

The expressions of the influence coefficients of the two matrices $\boldsymbol{C D}$ and $\boldsymbol{C S}$ can be found in [11].

The dynamic and kinematic free-surface conditions are linearized at the incident free surface elevation level $z=\eta^{I}$ :

$$
\begin{aligned}
\frac{D_{0} \phi^{P}}{D t}= & -\dot{\phi}^{I}-g\left(\eta^{I}+\eta^{P}\right)-\frac{1}{2} \boldsymbol{\nabla} \phi^{I} \cdot \boldsymbol{\nabla} \phi^{I} \\
& -\left(\boldsymbol{\nabla} \phi^{I}-\boldsymbol{v}_{\boldsymbol{m e s h}}\right) \cdot \boldsymbol{\nabla} \phi^{P} \\
& -\eta^{P}\left(\frac{\partial \dot{\phi}^{I}}{\partial z}+\frac{\partial\left(\boldsymbol{\nabla} \phi^{I} \cdot \boldsymbol{\nabla} \phi^{I}\right)}{\partial z}\right)-v \phi^{P} \\
\frac{D_{0} \eta^{P}}{D t}= & -\dot{\eta}^{I}+\frac{\partial\left(\phi^{I}+\phi^{P}\right)}{\partial z}-\boldsymbol{\nabla} \phi^{I} \cdot \boldsymbol{\nabla} \eta^{I} \\
& -\nabla \phi^{P} \cdot \boldsymbol{\nabla} \eta^{I}-\left(\boldsymbol{\nabla} \phi^{I}-\boldsymbol{v}_{\boldsymbol{m}} \boldsymbol{s h \boldsymbol { h }}\right) \cdot \boldsymbol{\nabla} \eta^{P} \\
& +\eta^{P}\left(\frac{\partial^{2} \phi^{I}}{\partial z^{2}}-\frac{\partial\left(\boldsymbol{\nabla} \phi^{I} \cdot \boldsymbol{\nabla} \eta^{I}\right)}{\partial z}\right)-v \eta^{P}
\end{aligned}
$$

The no-flux condition through the body surfaces leads to the following slip condition for every node of the $j^{\text {th }}$ body mesh:

$$
\phi_{n}^{P}=-\phi_{n}^{I}+\left[\boldsymbol{v}_{\boldsymbol{j}}+\boldsymbol{\omega}_{\boldsymbol{j}} \times\left(\boldsymbol{x}-\boldsymbol{x}_{\boldsymbol{j}}\right)\right] \cdot \boldsymbol{n}
$$

Where $\boldsymbol{v}_{\boldsymbol{j}}, \boldsymbol{\omega}_{\boldsymbol{j}}, \boldsymbol{x}_{\boldsymbol{j}}$ and $\boldsymbol{x}$ are the linear velocity, the angular velocity, the position of the center of gravity of the $j^{t h}$ body and the position of the node.
The last assumption of the fluid-structure problem is to consider that the bodies are rigid.

The far field condition is:

$$
\left\{\begin{array}{l}
\phi^{P} \rightarrow 0 \\
\eta^{P} \rightarrow 0
\end{array} \text { when } \sqrt{x^{2}+y^{2}+z^{2}} \rightarrow \infty\right.
$$

Equations (8), (9), (10), (11) and (12) constitute the first Boundary Value Problem (BVP) to solve [11].

At $t=0 \mathrm{~s}$, the flow is assumed to be at rest. The initial condition on the scattered components is:

$$
\left\{\begin{array}{l}
\phi^{P}=0 \\
\eta^{P}=0
\end{array} \text { at } \mathrm{t}=0 \mathrm{~s}\right.
$$

In order to avoid generation of non-physical waves when the simulation starts, a ramp function is used:

$$
f(t)=\begin{gathered}
0 \text { when } t \leq T_{1} \\
1 \text { when } t \geq T_{2} \\
\left(\frac{1}{2}\left[1-\cos \left(\pi \frac{t-T_{1}}{T_{2}-T_{1}}\right)\right]\right. \text { otherwise }
\end{gathered}
$$

With $T_{1}$ and $T_{2}$ be the starting and final time of the ramp.

The pressure is given by the Bernoulli's equation:

$p=-\rho\left[\dot{\phi}^{I}+\dot{\phi}^{P}+\frac{1}{2}\left(\nabla \phi^{I} \cdot \boldsymbol{\nabla} \phi^{I}+2 \boldsymbol{\nabla} \phi^{I} \cdot \boldsymbol{\nabla} \phi^{P}\right)+g z\right]$

Hydrodynamic and hydrostatic loads for the $j^{\text {th }}$ body are calculated by integration of the pressure over the wetted body surface:

$$
\boldsymbol{F}_{\boldsymbol{j}}=\begin{gathered}
\iint_{S_{B_{j}}} p \boldsymbol{n} d S \\
\left.\iiint_{S_{B_{j}}} p\left[\left(\boldsymbol{x}-\boldsymbol{x}_{\boldsymbol{j}}\right) \times \boldsymbol{n}\right] d S\right)
\end{gathered}
$$

The time-derivative of the scattered velocity potential, $\dot{\phi}^{P}$, is computed by solving a second Boundary Value Problem [11]. The integral equation of the second BVP becomes:

$$
C S \dot{\phi}_{n}^{P}-C D \dot{\phi}^{P}=0
$$

The time-derivative of the velocity potential, $\dot{\phi}^{P}$, is known on the free surface from Eq. (9). The time-derivative of the normal-derivative of the velocity potential, $\dot{\phi}_{n}^{P}$, is known from the time-derivative of the body slip condition (written for every node of the $j^{\text {th }}$ body mesh):

$$
\dot{\phi}_{n}^{P}=-\dot{\phi}_{n}^{I}+C K_{j} \ddot{\eta}_{j}+Q_{j}
$$


Equation (18) includes a new unknown, the acceleration of the $j^{\text {th }}$ body, $\ddot{\boldsymbol{\eta}}_{j}$. It is written as a function of the Cardan angles $\left(\begin{array}{lll}\varphi_{j} & \theta_{j} & \psi_{j}\end{array}\right)$

$$
\boldsymbol{\eta}_{\boldsymbol{j}}=\left(\begin{array}{llllll}
x_{j} & y_{j} & z_{j} & \varphi_{j} & \theta_{j} & \left.\psi_{j}\right)^{T}
\end{array}\right.
$$

$Q_{j}$ is an extra-term resulting of the time-differentiation of Eq. (11).

The motion equation for the $j^{\text {th }}$ body is used to close the system of differential equations. It is written in an inertial earth fixed reference frame $\Sigma_{e}$ with its origin $O_{e}$ and the unit vectors $\left(\boldsymbol{x}_{e}, \boldsymbol{y}_{\boldsymbol{e}}\right)$ lying on the mean water free surface. $\boldsymbol{z}_{\boldsymbol{e}}$ is pointing upwards. The motion equation is written at the center of gravity of the $j^{\text {th }}$ body.

$$
M_{j} \ddot{\eta}_{j}=F_{j}=C T_{j} \dot{\phi}^{P}\left(B_{j}\right)+T_{j}^{h}
$$

The expressions of $\boldsymbol{C K}_{\boldsymbol{j}}, \boldsymbol{C} \boldsymbol{T}_{\boldsymbol{j}}, \boldsymbol{T}_{\boldsymbol{j}}^{\boldsymbol{h}}$ and $Q_{j}$ are given in [15]. $\dot{\boldsymbol{\phi}}^{P}\left(B_{j}\right)$ is the vector of the values of $\dot{\phi}^{P}$ for every node of the mesh of the $j^{\text {th }}$ body. Only hydrodynamic loads are considered in Eq. (20).

Equations (17), (18) and (20) form the second Boundary Value Problem to solve. The final system of equations is:

$$
\left\{\begin{array}{c}
\boldsymbol{C S} \dot{\boldsymbol{\phi}}_{n}^{P}-\boldsymbol{C D} \dot{\boldsymbol{\phi}}^{P}=\mathbf{0} \\
\dot{\phi}_{n}^{P}=-\dot{\phi}_{n}^{I}+\boldsymbol{C} \boldsymbol{K}_{j} \ddot{\eta}_{j}+Q_{j} \forall j \in[|1, N|] \\
{ }^{e} \boldsymbol{M}_{j} \ddot{\eta}_{j}={ }^{e} \boldsymbol{C T}_{j} \dot{\boldsymbol{\phi}}^{P}\left(B_{j}\right)+{ }^{e} \boldsymbol{T}_{j}^{h} \forall j \in[|1, N|]
\end{array}\right.
$$

Equation (21) is the monolithic fluid-structure coupling solved in $W S_{-} C N$.

\section{Remeshing algorithm}

$W S \_C N$ was initially developed for a single body. It uses a discretization with triangular panels. It includes a mesh generation capability for the creation of unstructured meshes based on the advance front method [16]. Mesh deformation techniques are used to avoid remeshing at each time step which is required because of the motion of bodies. A linear spring analogy approach is applied to deform the mesh of the floating bodies [17]. Regarding the free surface mesh, the deformation is based on a radial basis function approach [18]. Nevertheless, mesh deformation is not sufficient to ensure a robust simulation for long simulations (several tens of seconds), particularly for the case of multibody simulations. Indeed, the mesh can be significantly distorted in case of large amplitude relative motion, which leads to numerical errors. For instance, the computation of the gradient of the velocity potential or the wave elevation is sensitive to the good quality of the mesh. Thus, including a remeshing capability is necessary.

In a remeshing process, new nodes are created. Physical quantities are unknowns at the locations of the new nodes. They need to be interpolated. On the free surface mesh, the scattered component of both the velocity potential and the wave elevation must be known, for instance, to time-step the model by using the free surface boundary equations (9) and (10). An interpolation algorithm must be applied to find $\phi^{P}$ and $\eta^{P}$ at their new locations from their values at the former mesh nodes. Third-order polynomial b-splines are used in the interpolation scheme. below:

The structure of the remeshing algorithm is described

Generation of a new mesh using the advance front method;

For each node of the new mesh, search of the nearest node of the former mesh;

B-splines approximation of the perturbed velocity potential and the perturbed wave elevation based on the nearest node and the neighboring nodes in the former mesh and evaluation at the location of the new node.

Furthermore, a Gaussian filter is applied to the free surface mesh [19] in order to remove high-frequency waves generated during the simulation. High-frequency waves lead to numerical instabilities. The filter is used every five time steps [20].

\section{Description of the test cases}

The test cases presented by Watai et al. in [1] are used for the validation of the capability of WS_CN to deal with multiple interacting floating bodies.

They conducted experimental tests at the University of Sao Paulo with two cylinders (one moving with a large amplitude motion, the other one being fixed) in regular waves. Both cylinders are circular with a dimeter of $0.40 \mathrm{~m}$, a height of 0.36 $\mathrm{m}$ and a draft of $0.20 \mathrm{~m}$. When the cylinders are aligned with the y-axis, they are separated by $0.6 \mathrm{~m}$ (center-to-center). The first cylinder (named Body 1) is fixed while a harmonic motion is prescribed to the second cylinder (Body 2), located upstream of Body 1. Three wave probes (WP1, WP2 and WP3) are used. WP2 is in the middle between Body 1 and Body 2 when they are aligned with the incident waves. WP1, respectively WP2, is $0.7 \mathrm{~m}$ upstream, respectively downstream, of WP2. Figure 1 shows the locations of each element along with the definition of the global frame.

Watai et al. [1] considered four regular waves. They are listed in Table 1. For each regular wave, three frequencies of the harmonic motion of Body 2 were selected. The motion amplitude of Body 2 is fixed at $0.37 \mathrm{~m}$. Table 2 shows the twelve cases. 


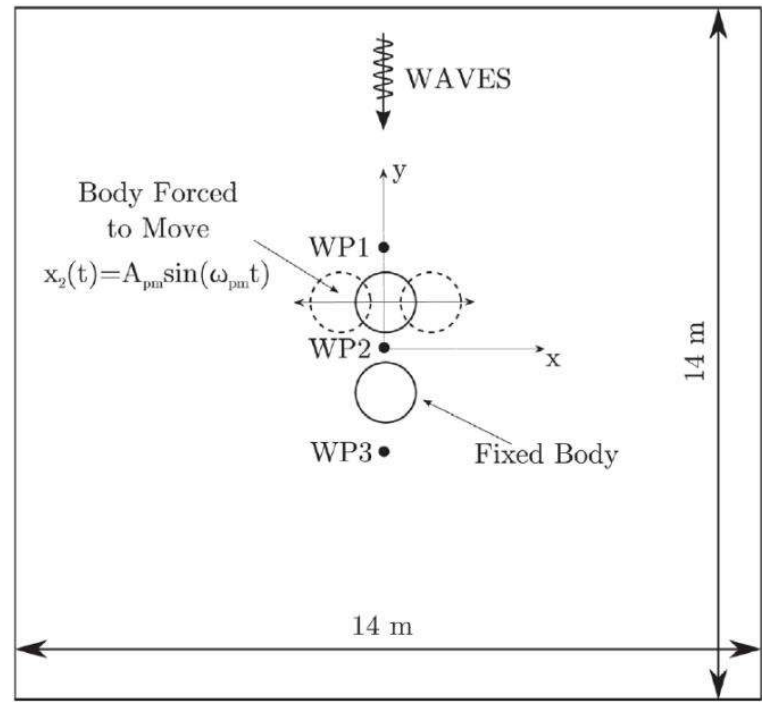

Figure 1: TOP VIEW OF THE SKETCH OF THE EXPERIMENTAL SET-UP (THE PICTURE IS TAKEN FROM [1])

\begin{tabular}{cccc}
\hline ID & $\omega(\mathrm{rad} / \mathrm{s})$ & $\lambda(\mathrm{m})$ & $\mathrm{A}(\mathrm{m})$ \\
\hline Reg 1 & 6.400 & 1.506 & 0.0115 \\
Reg 2 & 6.800 & 1.330 & 0.0100 \\
Reg 3 & 7.000 & 1.259 & 0.0095 \\
Reg 4 & 7.200 & 1.190 & 0.0090 \\
\hline
\end{tabular}

Table 1: REGULAR WAVE CHARACTERISTICS

\begin{tabular}{cccc}
\hline Case & Wave & $\omega_{p m}(\mathrm{rad} / \mathrm{s})$ & $A_{p m}(\mathrm{~m})$ \\
\hline 1 & Reg 1 & 0.427 & 0.37 \\
2 & Reg 1 & 0.213 & 0.37 \\
3 & Reg 1 & 0.107 & 0.37 \\
4 & Reg 2 & 0.453 & 0.37 \\
5 & Reg 2 & 0.227 & 0.37 \\
6 & Reg 2 & 0.113 & 0.37 \\
7 & Reg 3 & 0.467 & 0.37 \\
8 & Reg 3 & 0.233 & 0.37 \\
9 & Reg 3 & 0.117 & 0.37 \\
10 & Reg 4 & 0.480 & 0.37 \\
11 & Reg 4 & 0.240 & 0.37 \\
12 & Reg 4 & 0.120 & 0.37 \\
\hline
\end{tabular}

Table 2: CHARACTERISTICS OF THE TWELVE TEST CASES

\section{Numerical results}

A cylindrical domain is used for our simulations, with a radius of two wave lengths $(2 \lambda)$. One wave length is used for the damping beach. Figures 2 and 3 show the mesh of the domain for Case 1. Bodies are meshed at real scale. The ramp presented in Eq. (14) is applied for a wave period from the start of the simulation. The number of panels is approximately, respectively, 20000, 16000 and 13000 for, respectively, Cases 1,5 and 10 . The time step is $0.005 \mathrm{~s}$.

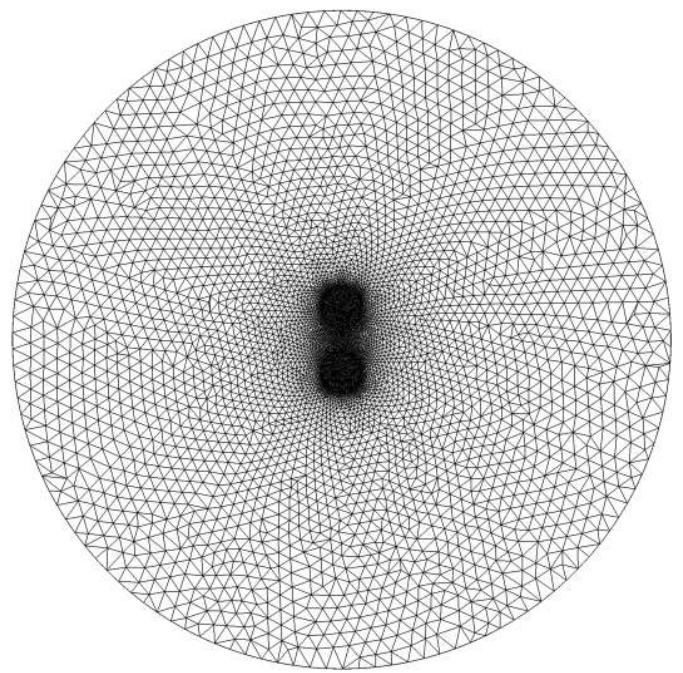

Figure 2: TOP VIEW OF THE MESH FOR CASE 1

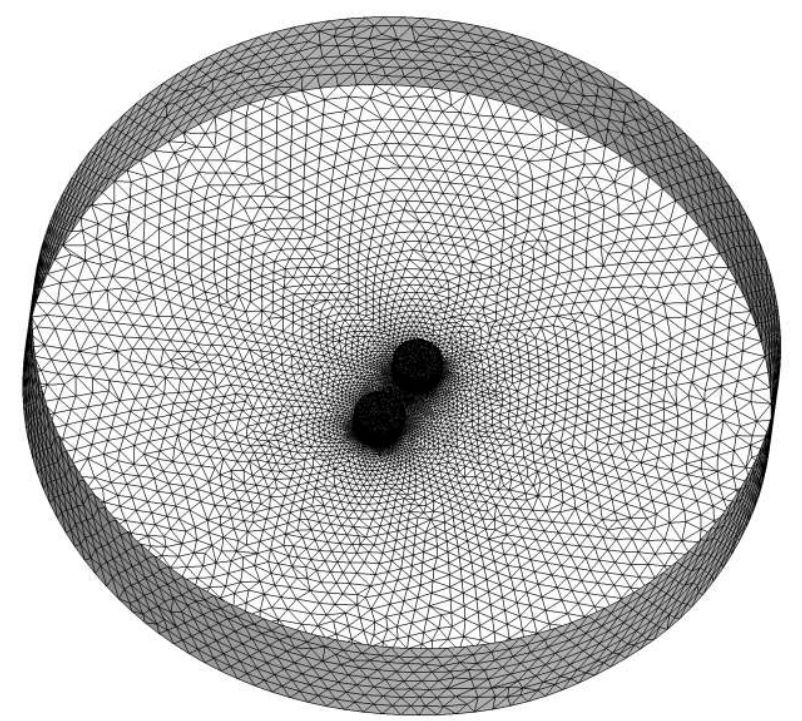

Figure 3: VIEW FROM BELOW OF THE MESH FOR CASE 1

Numerical results and experimental data of Watai et al. [1] are compared with respect to the wave elevations at the three wave probe locations and the hydrodynamic loads on Body 1. Hydrodynamic loads are computed from Eq. (16) after removal of the hydrostatic part of the Bernoulli Eq. (15). The results of Case 1 are presented on Fig. 4 for the loads on Body 1, Fig. 5 for the wave elevations and Fig. 6 for the loads on Body 2 . Cases 5 and 10 were also studied and comparisons are presented after digitization of the data of [1]. Hydrodynamic loads on Body 1 are presented for Case 5 on Fig. 7 and for Case 10 on Fig. 8. Experimental and numerical signals were synchronized $a$ posteriori, as the incident wave signals are not provided in [1]. 

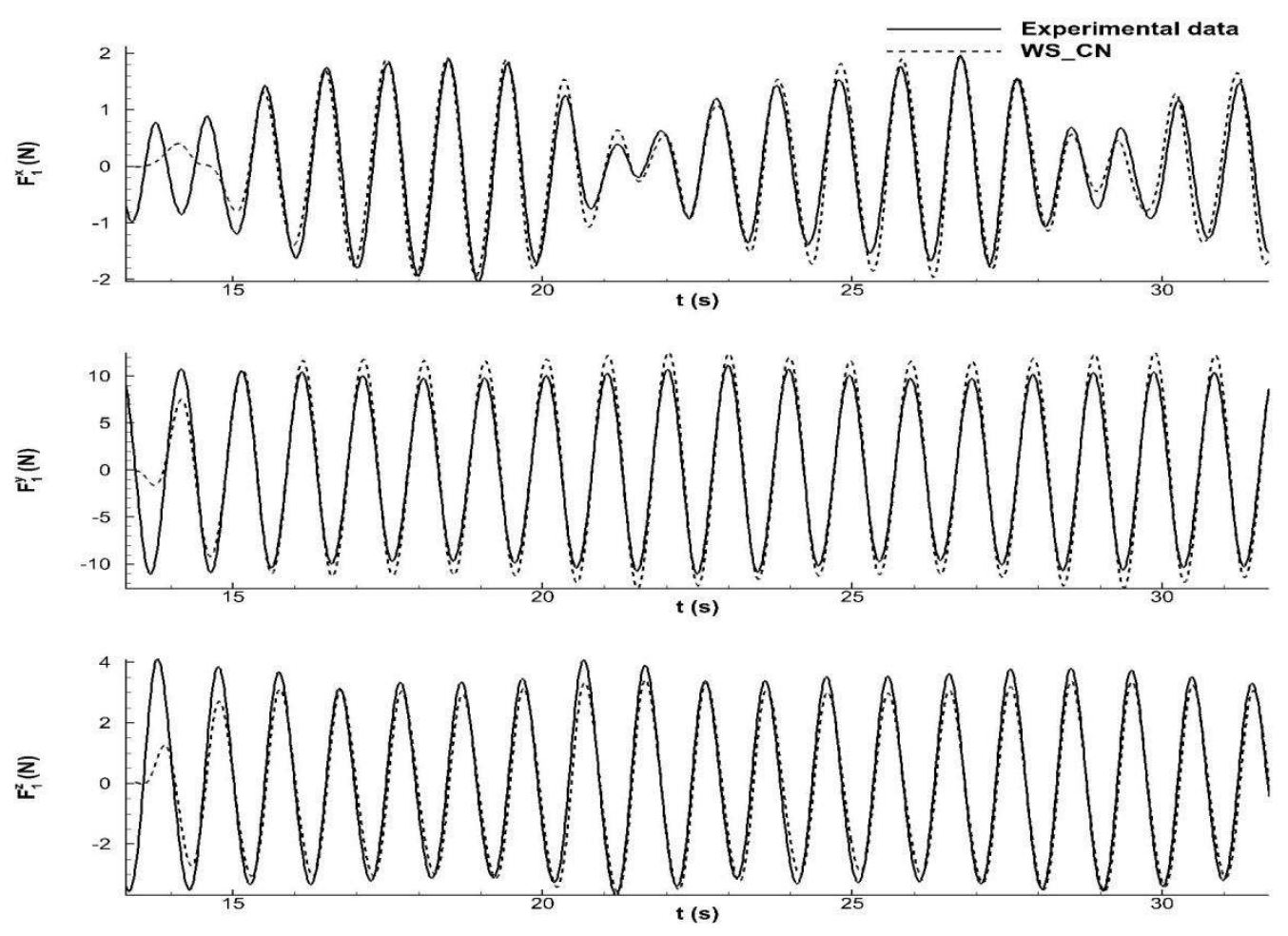

Figure 4: COMPARISON OF TIME SERIES OF THE HYDRODYNAMIC LOADS OF THE BODY 1 FROM NUMERICAL AND EXPERIMENTAL RESULTS FOR CASE 1
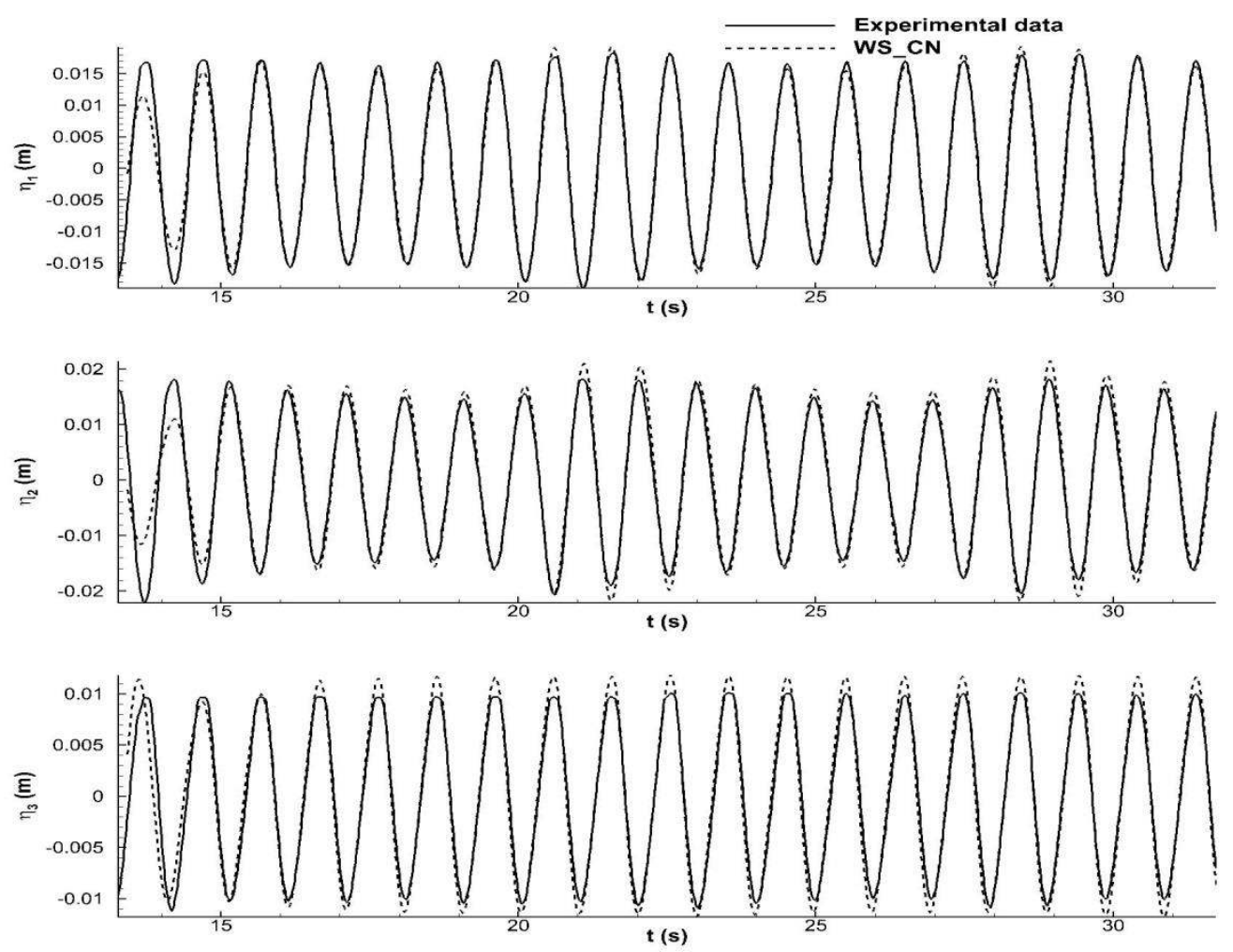

Figure 5: COMPARISON OF TIME SERIES OF THE WAVE ELEVATIONS AT WP1, WP2, AND WP3 FROM NUMERICAL AND EXPERIMENTAL RESULTS FOR CASE 1 

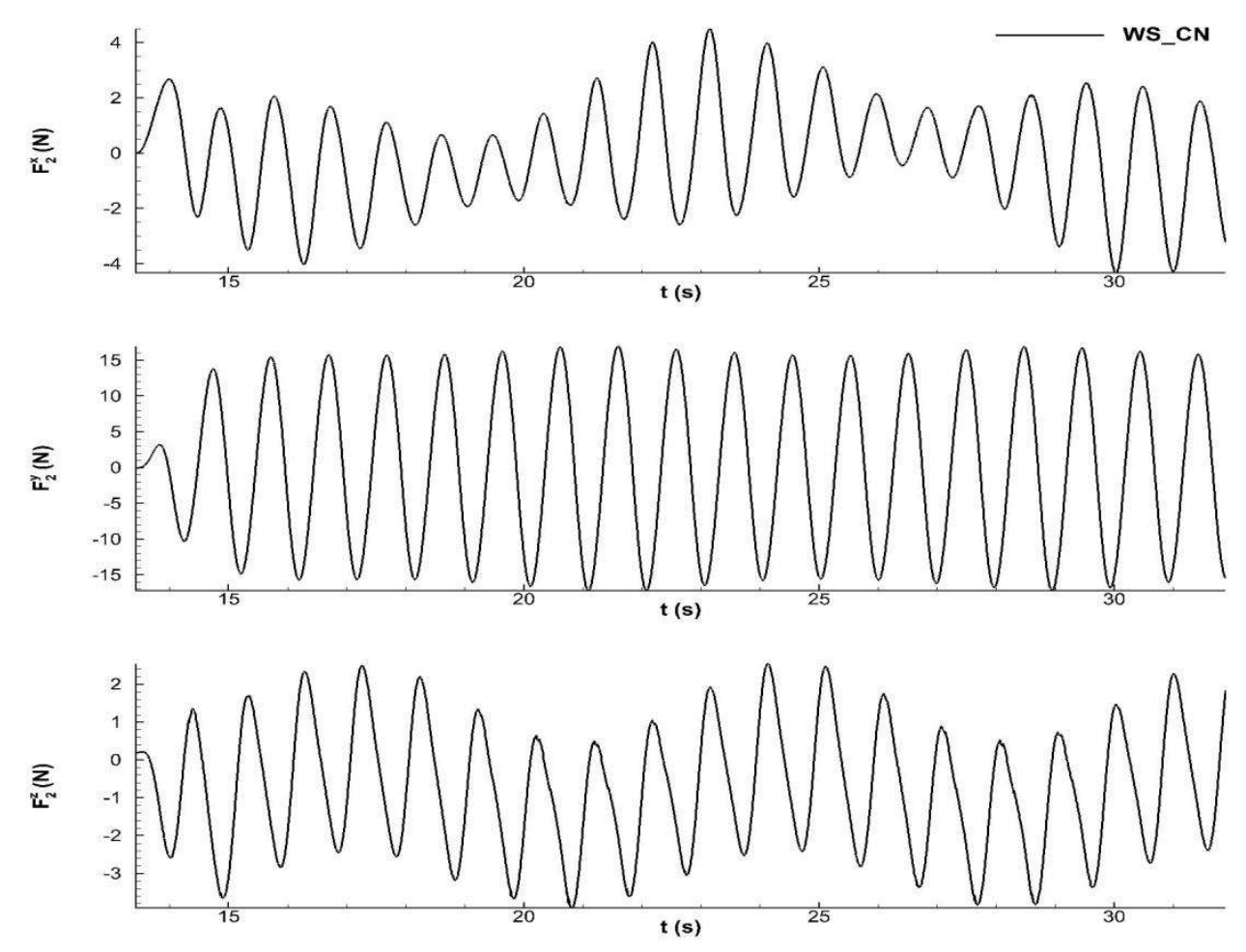

Figure 6: TIME SERIES OF THE HYDRODYNAMIC LOADS OF THE BODY 2 FROM NUMERICAL RESULTS FOR CASE 1
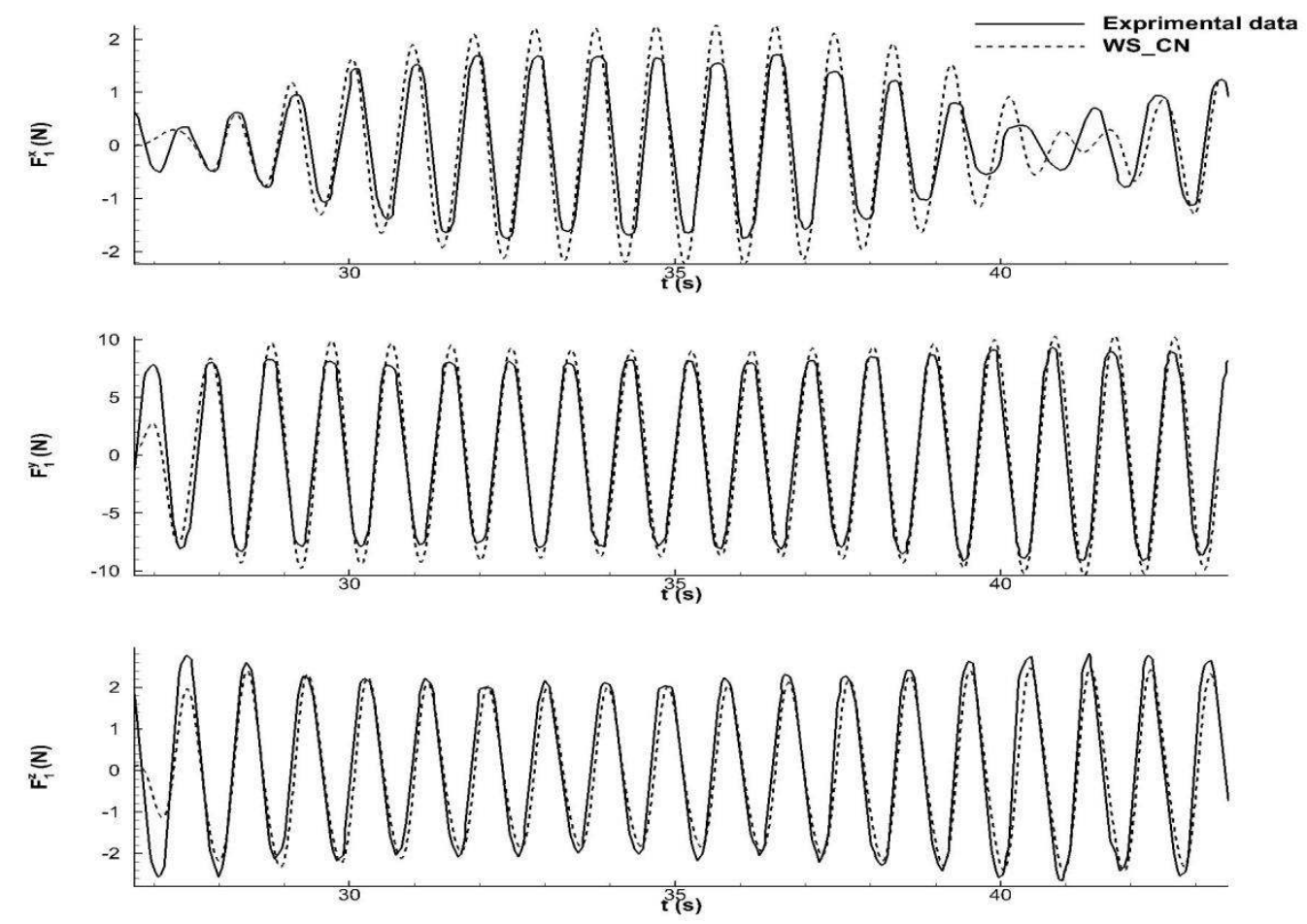

Figure 7: COMPARISON OF TIME SERIES OF THE HYDRODYNAMIC LOADS OF THE BODY 1 FROM NUMERICAL AND EXPERIMENTAL RESULTS FOR CASE 5 

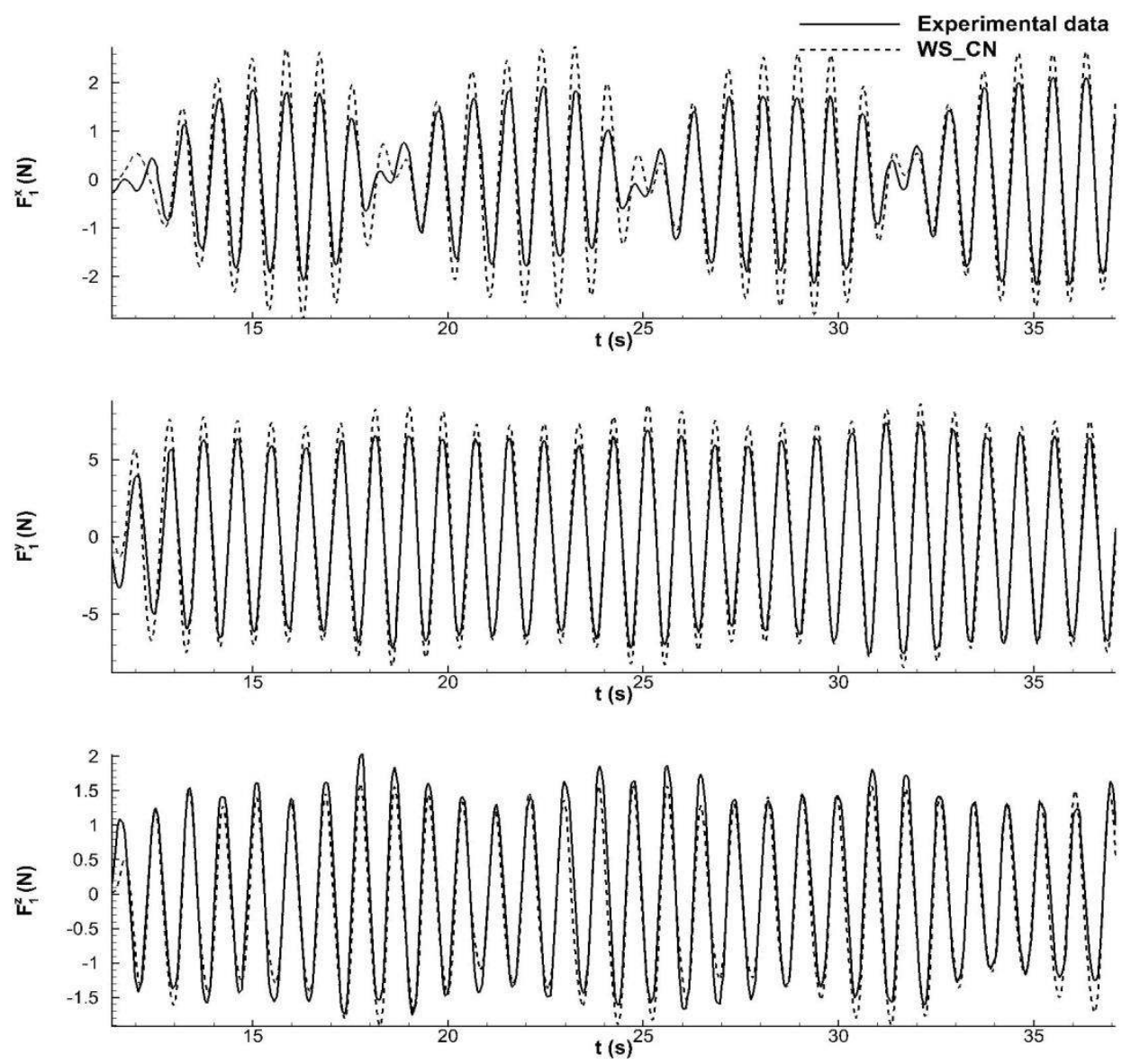

Figure 8: COMPARISON OF TIME SERIES OF THE HYDRODYNAMIC LOADS OF THE BODY 1 FROM NUMERICAL AND EXPERIMENTAL RESULTS FOR CASE 10

The time series of the hydrodynamic loads show an overall good agreement between the experimental data and $W S \_C N$. The modulation of the amplitude of $F_{1}^{x}$ on Fig 4, 7 and 8 is well captured. The low frequency oscillations match with the harmonic motion of Body 2. In the three test cases, one can note that WS_CN appears to overestimate the amplitude of the loads, especially for $F_{1}^{x}$ and $F_{1}^{y}$ while an underestimation is observed for $F_{1}^{Z}$. Regarding the wave elevation, the modulations in $\eta_{1}$ and $\eta_{2}$ on Fig. 5 appear in the numerical computations. The same overestimations, as the hydrodynamic loads, are noticed. Overestimations are also present in the work of Watai et al. [14] and are likely due to the main hypotheses of the solver (inviscid fluid, irrotational flow). Nevertheless Watai et al. needed much less panels (4010 panels in total for Case 1) than WS_CN (around 20000 panels in total for Case 1) so the method itself can also be responsible of the differences. The weakly nonlinear potential flow solver, used in this paper, requires meshing the water surface at incident wave elevation lever whereas Watai et al. use fully linear free surface conditions (water surface is water level at rest). In $W S_{-} C N$, mesh convergence requires much more panels. The frequent use of remeshing processes, because of the large relative motion, leads to a high number of interpolations of the physical quantity on the free surface (once per time step), deteriorating the accuracy, especially in case of small time steps. 
For sake of illustration, the wave pattern for Case 1 at $\mathrm{t}=$ $24.482 \mathrm{~s}$ is displayed on Fig. 9.

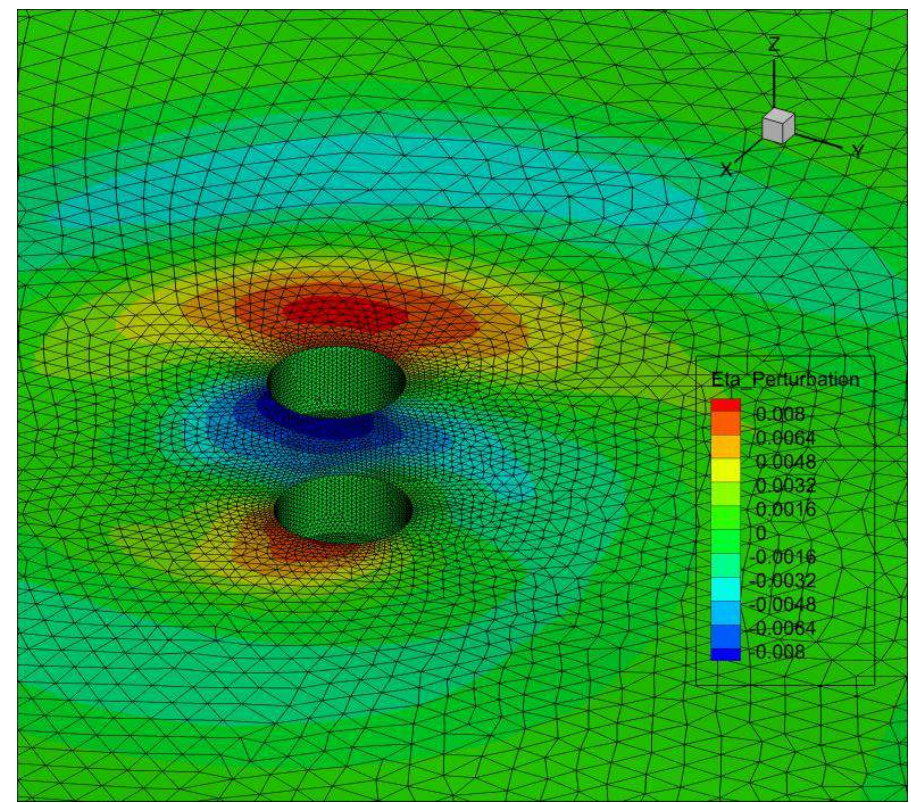

Figure 9: PERTURBATION COMPONENT OF THE WAVE PATTERN $\left(\boldsymbol{\eta}^{\boldsymbol{P}}\right)$ AT $\mathrm{t}=\mathbf{2 4 . 4 8 2}$ s FOR CASE 1

\section{PART II: SIMULATION OF THE BALLASTING OF A SPAR}

\section{Description of the operation}

In [13], a coupling between the weakly nonlinear potential flow solver of LHEEA and InWave is presented for the case of a single floating body as root of the multibody system. It uses a tight coupling method. The coupling has been extended to multibody systems [21]. There are no limitations on the location of the floating bodies in the multibody system. This new numerical tool is applied to the simulation of the ballasting of a spar.

The spar is a cylinder. It is kept horizontal at the beginning of the experiment with a cable (Fig. 10a). Then, the cable is unwound (Fig. 10b) until the spar reaches a vertical position (Fig. 10c). Thus, the modeling of this experimental setup allows having to deal with an articulated multibody system (cable and floating cylinder) and a floating body with an important deformation of the wetted surface (reason for using the weak-scatterer code). The comparison between the numerical results of the coupling and the experimental data aims at assessing the performance and the consistency of the coupling theory.
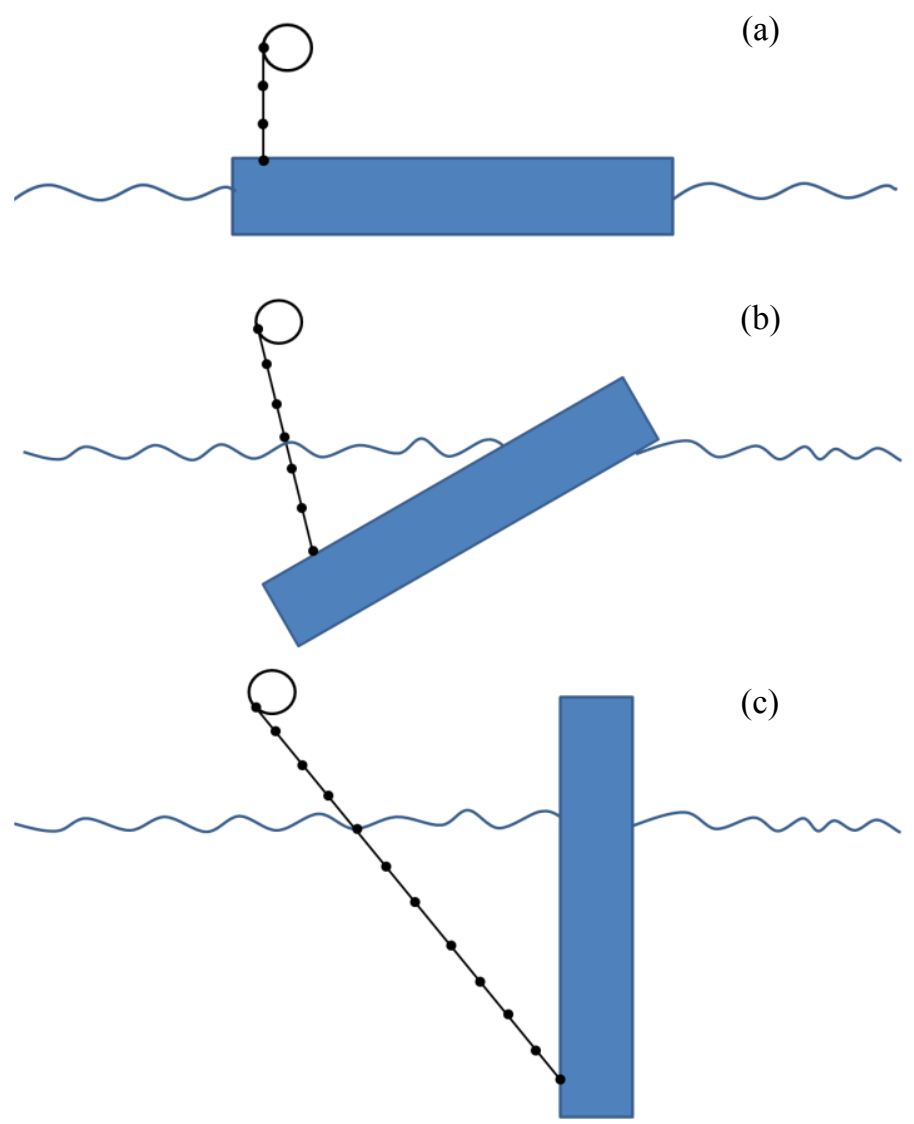

Figure 10: SKETCHES OF THE PHASES OF THE BALLASTING

\section{Experimental setup}

The cylindrical buoy (illustrated in Fig. 11) which represents the spar, is made of three parts: an external PVC pipe, a lead weight and a threaded rod. Table 3, respectively Table 4, shows the geometrical characteristics, respectively the mass and the inertia, of the buoy. Inertias are given with respect to an axis perpendicular to the axis of revolution of the buoy and passing by the reference point. At its equilibrium position the buoy is vertical (Fig. 10c), therefore at the initial time (Fig. 10a) the floating cylinder is maintained by the use of the cable. This cable is stiff (negligible elasticity). The mass of the buoy is constant through the operation. 

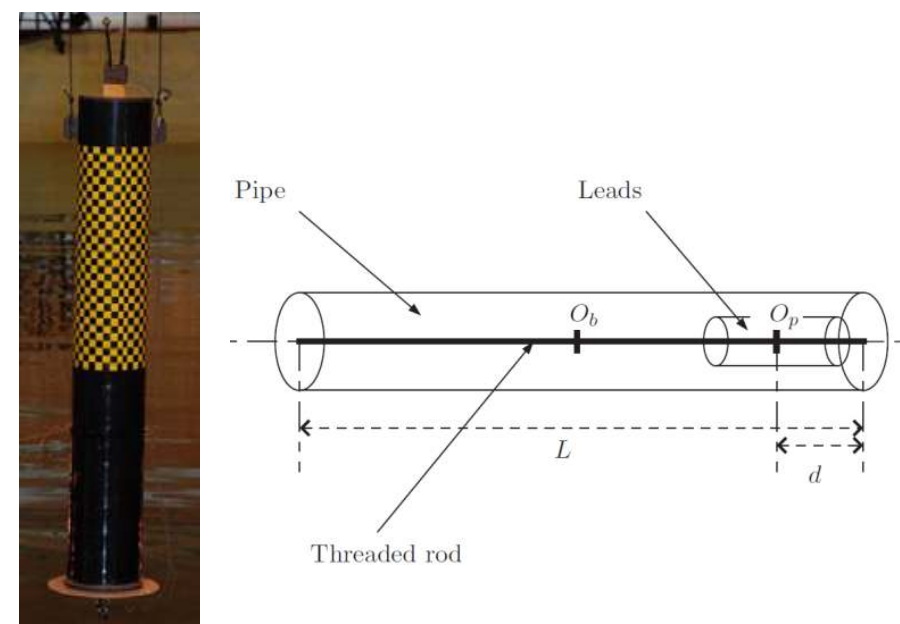

Figure 11: PICTURE AND SKETCH OF THE BUOY

\begin{tabular}{ccc}
\hline Length & Symbol & Value $(\mathrm{m})$ \\
\hline Buoy length & $\mathrm{L}$ & 1.32 \\
Buoy diameter & $\mathrm{D}$ & 0.20 \\
Leads location & $\mathrm{d}$ & 0.13 \\
\hline
\end{tabular}

Table 3: GEOMETRICAL CHARACTERISTICS OF THE BUOY

\begin{tabular}{cccc}
\hline Element & Mass $(\mathrm{kg})$ & Inertia $\left(\mathrm{kg} \cdot \mathrm{m}^{2}\right)$ & $\begin{array}{c}\text { Reference } \\
\text { point }\end{array}$ \\
\hline Pipe & 10 & 1.5 & $O_{b}$ \\
Leads & 16 & 0.058 & $O_{p}$ \\
Threaded rod & 2 & 0.27 & $O_{b}$ \\
\hline
\end{tabular}

Table 4: MASS AND INERTIA OF EACH PART OF THE BUOY

At time of writing, the experiments are being conducted in the shallow water wave basin (Fig. 12) of LHEEA. Its dimensions are $20 \mathrm{~m} \times 9.5 \mathrm{~m}$ with a water depth of $1 \mathrm{~m}$.

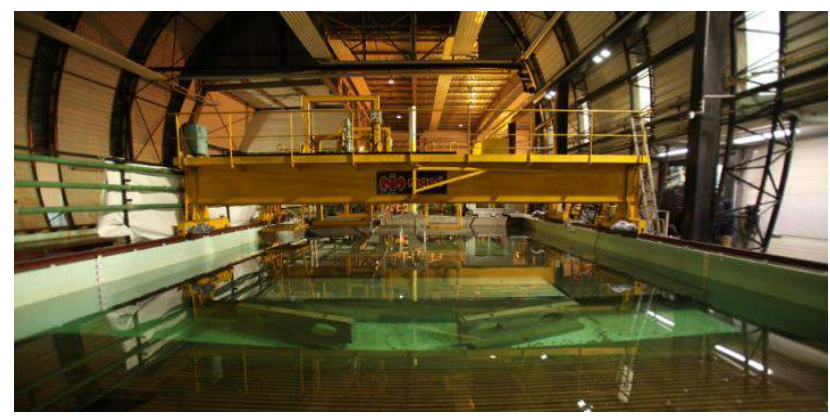

Figure 12: SHALLOW WATER BASIN AT LHEEA

\section{Numerical modeling}

The multibody solver In Wave is used to simulate both the buoy and cable dynamics. The cable is discretized into cable elements. The multibody approach used in InWave is equivalent to the classical low-order lumped mass theory [13]. Bending and torsion are neglected, only axial tension and damping are considered. The lowering of the cable is achieved by increasing the unstretched length of the cable element connected to the winding mechanism [13].

The multibody motion equation without taking into account the hydrodynamic loads is [22]:

$$
\left(\begin{array}{ll}
H_{11} & H_{12} \\
H_{21} & H_{22}
\end{array}\right)\left(\begin{array}{c}
0 \\
\dot{V}_{0} \\
\ddot{q}
\end{array}\right)=\left(\begin{array}{c}
0_{6 \times 1} \\
\Gamma
\end{array}\right)-\left(\begin{array}{c}
C_{1} \\
C_{2}
\end{array}\right)
$$

The first row is the motion equation for the base, which is the root body for the multibody system. The second row is the motion equation for the other bodies.

This equation is coupled with the second Boundary Value Problem in order to solve, in a tightly coupled manner, both the accelerations $\left(\begin{array}{c}{ }^{0} \dot{\boldsymbol{V}}_{\mathbf{0}} \\ \ddot{\boldsymbol{q}}\end{array}\right)$ and the scattered component of the time-differentiation of the velocity potential $\dot{\phi}^{P}$ [21].

Figure 13 shows the mesh of the buoy and a 3 -element cable at the beginning of the simulation.

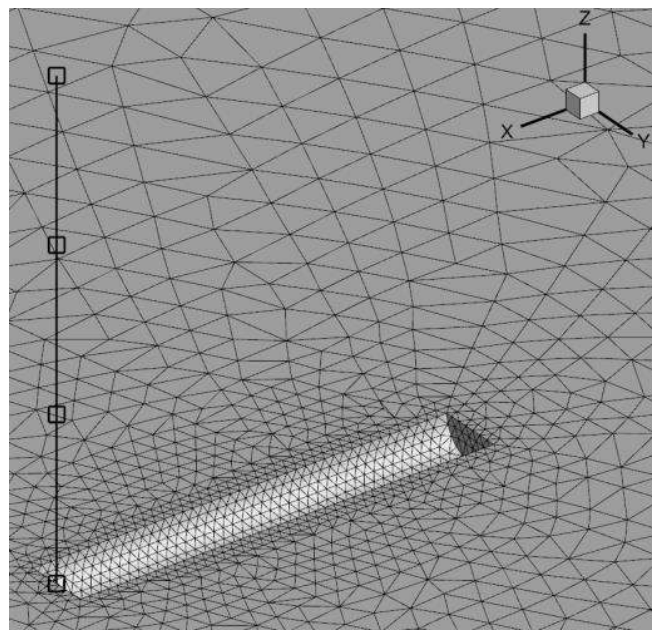

Figure 13: MESH OF THE CYLINDRICAL BUOY AND A 3-ELEMENT CABLE 


\section{CONCLUSIONS}

First, this paper presented the extension of a weakscatterer assumption based potential flow solver, $W S \_C N$, to multibody simulations. A validation has been achieved by comparing numerical results to the experimental data of Watai et al. [1]. It shows a good agreement between the numerical and experimental results in terms of hydrodynamic loads and wave elevations.

A second validation is proposed. It consists in the ballasting of a spar. Comparison results will be presented at the conference.

\section{ACKNOWLEDGMENTS}

The authors acknowledge R. A. Watai and A. N. Simos for having shared their experimental data and answered our questions. The authors also acknowledge Maxime Philippe for his participation at the beginning of this project.

\section{REFERENCES}

[1] R. Watai, F. Ruggeri, A. Simos, A new time domain Rankine panel method for simulations involving multiple bodies with large relative displacements, Applied Ocean Research, 59, pp 93-114, 2016.

[2] DNV-RP-H103, Modelling and analysis of marine operations, DNV, 2011.

[3] OrcaFlex Manual version 9.1a, Orcina, 2007.

[4] DeepLines 4.5 Theory Manual, Principia, 2011.

[5] Time domain analysis of multi body dynamics for offshore operations: aNySIM, MARIN.

[6] SIMO - Theory manual Version 3.6, rev: 2, MARINTEK, 2009.

[7] R. Watai, A time-domain boundary elements method for the seakeeping analysis of offshore systems, $\mathrm{PhD}$ thesis, 2015.

[8] J.C. Gilloteaux, Mouvements de grande amplitude d'un corps flottant en fluide parfait. Application à la récupération de l'énergie des vagues (in french), $\mathrm{PhD}$ thesis, 2007.

[9] M. Hannan, Numerical simulation of submerged payload coupled with crane barge in waves, $\mathrm{PhD}$ thesis, 2014.

[10] J. Pawlowski, A nonlinear theory of ship motion in waves, $19^{\text {th }}$ Symposium on Naval Hydrodynamics, 1991.

[11] L. Letournel, Développement d'un outil de simulation numérique basé sur l'approche weak-scatterer pour l'étude des systèmes houlomoteurs en grands mouvements (in french), $\mathrm{PhD}$ thesis, 2015.

[12] C. Chauvigné, L. Letournel, A. Babarit, G. Ducrozet, P. Bozonnet, J-C. Gilloteaux, P. Ferrant, Progresses in the development of a weakly-nonlinear wave body interaction model based on the weakscatterer approximation, Proceedings of the ASME 2015 34rd International Conference on Ocean, Offshore and Artic Engineering, St. John's, Newfoundland, Canada, 2014.

[13] P.-Y. Wuillaume, F. Rongère, A. Babarit, M. Philippe, P. Ferrant, Development and adaptation of the Composite Rigid Body Algorithm and the WeakScatterer approach in view of the modeling of marine operations, Proceeding of the $23^{\text {ème }}$ Congrès Français de Mécanique, Lille, France, 2017.

[14] A. Combourieu, M. Philippe, F. Rongère, A. Babarit, InWave: A new flexible design tool dedicated to wave energy converters, Proceedings of the ASME 2014 $33^{\text {rd }}$ International Conference on Ocean, Offshore and Artic Engineering, San Francisco, United States, 2014.

[15] Chauvigné C., Tenue à la mer d'un flotteur animé de grands mouvements pour les Energies Marines Renouvelables (in french), $\mathrm{PhD}$ thesis, 2016.

[16] C. Chauvigné, L. Letournel, A. Babarit, J.-C. Gilloteaux, P. Bozonnet, G. Ducrozet, P. Ferrant, Automatic mesh generation and mesh morphing methods for a weakly non-linear flow model based on the weakscatterer approximation (in preparation), 2017.

[17] Selim MM, Koomullil RP, Mesh deformation approaches - a survey, Journal of Physical Mathematics, 2016.

[18] A. de Boer, M. van der Schoot, H. Bijl, Mesh deformation based on radial basis function interpolation, Computers \& structures, 85, 784-795, 2007.

[19] L. Letournel, Quantification de l'effet du lissage Gaussien ajouté dans le code WS (internal report, in french), LHEEA, 2017.

[20] Y.-L. Shao, O. M. Faltinsen, Fully-nonlinear wave-current-body interaction analysis by a harmonic polynomial cell method, Journal of Offshore Mechanics and Artics Engineering, 136, 2014.

[21] P.-Y. Wuillaume, Coupling of multibody theories and a weak-scatterer approach (internal report), LHEEA, 2017.

[22] F. Rongère, A. H. Clément, Systematic dynamic modeling and simulation of multibody offshore structures: application to wave energy converters, Proceeding of the $32^{\text {nd }}$ International Conference on Ocean, Offshore and Artic Engineering, Nantes, France, 2013. 